\title{
A novel 3D template for mandible and maxilla reconstruction: Rapid prototyping using stereolithography
}

\author{
Samir Kumta, Monica Kumta ${ }^{1}$, Leena Jain, Shrirang Purohit, Rani Ummul \\ Department of Plastic and Reconstructive Surgery, Lilavati Hospital and Research Centre, ${ }^{1}$ Department of Chemical \\ Engineering, Institute of Chemical Technology, Mumbai, Maharashtra, India
}

Address for correspondence: Dr. Leena Jain, No. 301, C Wing, Dheeraj Presidency, MG Road, Kandivali West, Mumbai - 400067, Maharashtra, India. E-mail: Link.jain@gmail.com

\section{ABSTRACT}

Introduction: Replication of the exact three-dimensional (3D) structure of the maxilla and mandible is now a priority whilst attempting reconstruction of these bones to attain a complete functional and aesthetic rehabilitation. We hereby present the process of rapid prototyping using stereolithography to produce templates for modelling bone grafts and implants for maxilla/mandible reconstructions, its applications in tumour/trauma, and outcomes for primary and secondary reconstruction. Materials and Methods: Stereolithographic template-assisted reconstruction was used on 11 patients for the reconstruction of the mandible/maxilla primarily following tumour excision and secondarily for the realignment of post-traumatic malunited fractures or deformity corrections. Data obtained from the computed tomography (CT) scans with 1-mm resolution were converted into a computer-aided design (CAD) using the CT Digital Imaging and Communications in Medicine (DICOM) data. Once a CAD model was constructed, it was converted into a stereolithographic format and then processed by the rapid prototyping technology to produce the physical anatomical model using a resin. This resin model replicates the native mandible, which can be thus used off table as a guide for modelling the bone grafts. Discussion: This conversion of two-dimensional (2D) data from CT scan into 3D models is a very precise guide to shaping the bone grafts. Further, this CAD can reconstruct the defective half of the mandible using the mirror image principle, and the normal anatomical model can be created to aid secondary reconstructions. Conclusion: This novel approach allows a precise translation of the treatment plan directly to the surgical field. It is also an important teaching tool for implant moulding and fixation, and helps in patient counselling.

\section{KEY WORDS}

Rapid prototyping; stereolithography; three dimensional (3D) template

\begin{tabular}{|l|l|}
\hline \multicolumn{2}{|c|}{ Access this article online } \\
\hline Quick Response Code: & Website: \\
\hline & www.ijps.org \\
\cline { 2 - 2 } & Dol: \\
\hline
\end{tabular}

This is an open access article distributed under the terms of the Creative Commons Attribution-NonCommercial-ShareAlike 3.0 License, which allows others to remix, tweak, and build upon the work non-commercially, as long as the author is credited and the new creations are licensed under the identical terms.

For reprints contact: reprints@medknow.com

How to cite this article: Kumta S, Kumta M, Jain L, Purohit S, Ummul R. A novel 3D template for mandible and maxilla reconstruction: Rapid prototyping using stereolithography. Indian J Plast Surg 2015;48:263-73. 


\section{INTRODUCTION}

steocutaneous free flap reconstruction is the primary treatfment modality for defects of the mandible and midface following tumour extirpation or post-traumatic defects.

In the absence of a model, plates are bent intraoperatively in a standard fashion to conform to the native mandible. Bone graft and fixation plates have to be reshaped during the operation by trial and error, which is often a time-consuming procedure. The bone graft must be of the exact dimensions and shape as the defect to assure a precise three-dimensional configuration of the reconstructed mandible. Otherwise, the postoperative surgical outcome often shows an unsatisfactory 3D mandible shape and projection as well as disturbed function, thereby affecting the patient's quality of life.

To improve the 3D reconstruction of the facial skeleton, we present the use of a reverse printing or rapid prototyping technology method called stereolithography. We present in this paper a detailed description of the process of stereolithography and its applications in various cases for primary and secondary reconstruction.

\section{MATERIALS AND METHODS}

The flow chart below is a schematic of the concept of creating a 3D model from 2D data obtained from computed tomography (CT) scans and it involves two main steps.

The first step is to convert the 2D data of the head and neck CT scan of the patient (having maxilla or mandible involvement requiring resection and reconstruction) into a 3D data-using software called MIMICS (Materialise NV, Belgium). Once the 3D computerised data are ready, there is a computer-assisted design (CAD).

Once the CAD is ready, by 3D printing technology (also called reverse printing or rapid prototyping) the model is prepared using resin and laser.

CAD gives the input of each layer of the model to be manufactured at the level of each millimeter. The model is made from resin and laser is used to manufacture it layer by layer.

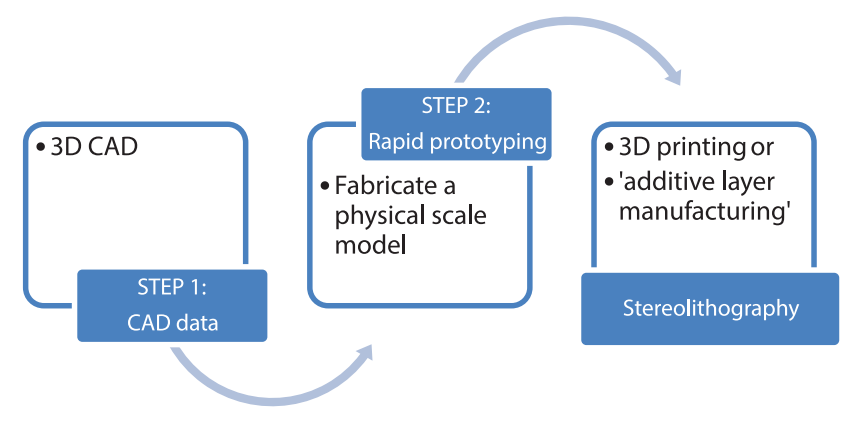

Step 1: CAD generation..$^{[1,2]}$

Here, the two-dimensional (2D) data from CT scans are converted into a 3D model.

In the sequence of images of CT scan, representing the various sections, the anatomical structures can be identified on the basis of the grey level of the pixels. One-millimeter sections are used to format the 3D virtual models (CAD). These anatomical regions are contoured using segmentation algorithms, and the 3D structure is reconstructed by generating skinning surfaces that join the resulting profiles, leading to very accurate $3 \mathrm{D}$ models. One such software to construct a 3D model form 2D data is called MIMICS. MIMICS is an acronym for 'Materialise Interactive Medical Image Control System'. We used MIMICS to create 3D surface models from stacks of 2D image data.

This 3D planning software allows an undistorted visualisation of the maxilla and mandible in four views: Axial, cross-sectional, panoramic and 3D reformatted data. The 3D files are represented in the stereolithographic (STL) format.

To summarise, the conversion of 2D data to 3D data is by the following:

Input formats: Digital Imaging and Communications in Medicine (DICOM), TIFF, JPEG, BMP and RAW

Output formats (3D): STL, VRML, PLY and DXF

Step 2: Rapid prototyping using stereolithography to construct the anatomical model made of resin..$^{[3-5]}$

Started as early as in 1986, 3D printing was available at the industrial level but this technology has only recently entered the consumer space. It was called 
rapid prototyping mainly because it was originally conceived as a faster and more cost-effective method for creating prototypes for product development within the industry. After a prototype was created, it was then sent for mass production. The colloquial name is ' $3 \mathrm{D}$ printing', implying the extension of $2 \mathrm{D}$ design into 3D model making. Rapid prototypying, another name for 3D printing, can also be used to produce solid, physical, hard copies of human anatomy from 3D computer data.

Rapid prototyping has also been referred to as solid free-form manufacturing, computer-automated manufacturing, additive manufacturing and layered manufacturing.

The American Society for Testing and Materials (ASTM) group developed a set of standards that classify the Additive Manufacturing processes into 7 categories, as set out in 2010:

1. Vat photopolymerisation.

2. Material jetting.

3. Binder jetting.

4. Material extrusion.

5. Powder bed fusion.

6. Sheet lamination.

7. Directed energy deposition.

\section{Vat photopolymerisation}

A 3D printer based on this method uses a container filled with photopolymer resin in liquid form which is then hardened with ultraviolet (UV) light source layer by layer. The most commonly used technology in this process is stereolithography (SLA). This technique was invented in 1986 by Charles Hull. This technology employs a vat of liquid UV-curable photopolymer resin, and a UV laser is used to build the object's layers one by one.

After the pattern has been traced, the SLA's elevator platform descends by a distance equal to the thickness of a single layer, typically 0.05-0.15 mm (0.002-0.006"). Then, a resin-filled blade sweeps across the cross section of the part, re-coating it with fresh material. On this new liquid surface, the subsequent layer pattern is traced, joining the previous layer. The complete 3D object is formed by this project. Stereolithography requires the use of supporting structures which serve to attach the part to the elevator platform and to hold the object because it floats in the basin filled with liquid resin. These are removed manually after the object is finished.

Other technologies using vat photopolymerisation are the new ultrafast continuous liquid interface production (CLIP) and the marginally used, older film transfer imaging and solid ground curing.

\section{Material jetting}

In this process, material is applied in droplets through a small-diameter nozzle, similar to the way a common inkjet paper printer works, but it is applied layer by layer to a build platform, making a 3D object, and then hardened by UV light [Figure 1].

\section{Binder jetting}

With binder jetting, two materials are used: Powder base material and a liquid binder. In the build chamber, powder is spread in equal layers and binder is applied through jet nozzles that 'glue' the powder particles in the shape of a programmed 3D object. The finished object is 'glued together' by binder remaining in the container with the powder base material. This technology was first developed at the Massachusetts Institute of Technology in 1993.

\section{Material extrusion}

The fused deposition modelling (FDM) technology, also called fused filament fabrication (FFF), developed in the late 80 s, works using a plastic filament or metal wire which is unwound from a coil, supplying material to an extrusion nozzle which can turn the flow on and off. The nozzle is heated to melt the material and can be moved in both horizontal and vertical directions by a

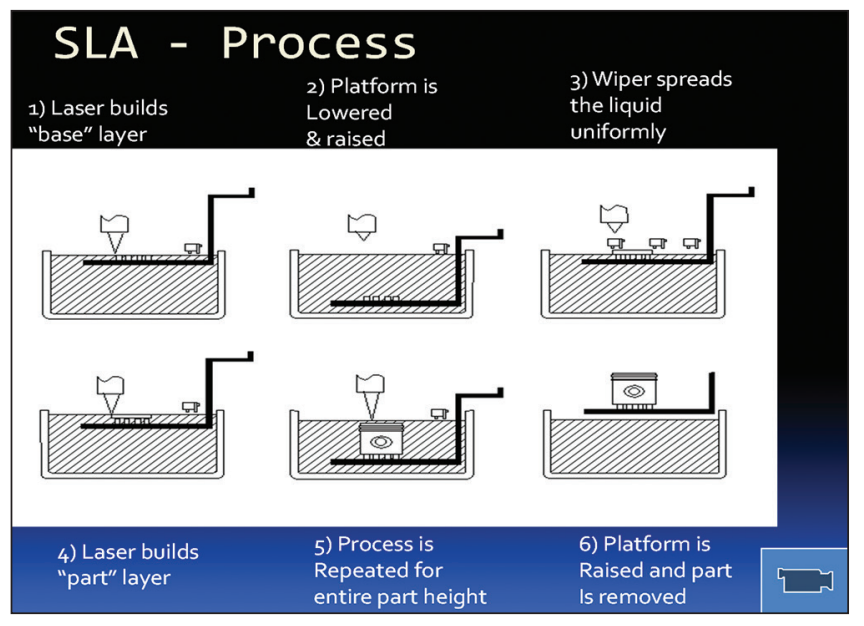

Figure 1: Schematic process of stereolithographic model construction (with permission from Mr. Paresh Bheda) 
numerically controlled mechanism, directly controlled by a computer-aided manufacturing (CAM) software package. The object is produced by extruding melted material to form layers, as the material hardens immediately after extrusion from the nozzle. This technology is most widely used with two plastic filament material types: Acrylonitrile butadiene styrene (ABS) and PLA (polylactic acid) but many other materials are available ranging in properties from wood-filed to conductive and flexible, among others.

\section{Powder bed fusion}

The most commonly used technology in this category is selective laser sintering (SLS), where high-power laser is used to fuse small particles of plastic, metal, ceramic or glass powders into a mass that has the desired 3D shape. The laser selectively fuses the powdered material by scanning the cross sections (or layers) generated by the 3D modelling program on the surface of a powder bed. After each cross section is scanned, the powder bed is lowered by one layer thickness. Then a new layer of material is applied on top and the process is repeated until the object is completed.

\section{Sheet lamination}

Sheet lamination involves material in sheets, which is bound together with external force. Sheets can be metal, paper or a form of polymer. Metal sheets are welded together by ultrasonic welding in layers and then computer numeric control (CNC)-milled into a proper shape. Paper sheets can be used also, but they are glued by adhesive glue and cut in shape by precise blades.

\section{Directed energy deposition}

This process is mostly used in the high-tech metal industry and in rapid manufacturing applications. The 3D printing apparatus is usually attached to a multi-axis robotic arm and consists of a nozzle that deposits metal powder or wire on a surface and an energy source (laser, electron beam or plasma arc) that melts it, forming a solid object.

Stereolithography, considered to be one of the first rapid prototyping techniques, was developed by 3D Systems (Valencia, CA, USA). The company was founded in 1986.

Stereolithography is the most well-known and used rapid-prototype technique. Stereolithography (SLA, also known as optical fabrication, photo-solidification, solid free-form fabrication, solid imaging, and resin printing) is a $3 \mathrm{D}$ printing technology used to produce models or patterns.

A rapid prototyping machine that uses the principle of stereolithography is employed to fabricate the stereolithographic models. The SLA consists of a vat that contains a liquid photo-polymerised resin. A laser is mounted on top of the vat and moves in sequential cross-sectional increments of $1 \mathrm{~mm}$, which correspond to the slice intervals specified during the CT formatting procedure.

The laser polymerises the surface layer of the resin on contact. Once the first slice is completed, a mechanical table that is immediately below the surface moves down $1 \mathrm{~mm}$, carrying with it the previously polymerised resin layer of the model. ${ }^{[5-7]}$ The laser now polymerises the next layer, which is above the previously polymerised layer. In this manner, a complete stereolithographic model of the patient's jaw can be created layer by layer with millimeter precision.

Approximately $80 \%$ of the total polymerisation is completed in the vat; the remaining $20 \%$ can be completed in a conventional UV light curing unit. The resin used to fabricate the model is stereocol. This is a photo-polymerised resin, United States Food and Drug Administration (FDA)-approved. The templates can be sterilised by using extremely common techniques such as applying low-temperature steam and formaldehyde at $80^{\circ} \mathrm{C}$, without loss of properties.

The basic methodology of the stereolithographic rapid prototyping technique can be summarised as follows:

1. A CAD model is constructed and converted to STL format.

2. The RP machine processes the STL file by creating sliced layers of the model.

3. The first layer of the physical model is created. The model is then lowered by the thickness of the next layer, and the process is repeated until completion of the model.

4. The model and any supports are removed. The surface of the model is then finished and cleaned.

The anatomical model of the maxilla or the mandible is thus created, and the surgeon then plans the resection and reconstruction. In cases where a hemi-mandible (to 
be reconstructed) is distorted due to tumour or trauma, the opposite normal hemi-mandible is used as a template and, using its mirror image, the affected hemi mandible model is thus created. In this way, a functionally precise reconstruction can be planned even in the absence of the native template.

On the model, the extent of resection of the bone is marked and the defect template is now ready. Using the same technology, the reconstruction template is constructed with accuracy. In accordance with this reconstruction template, we plan the number and angle of osteotomies required in the fibula and also plan the contouring of the reconstruction plate and anchorage sites.

The geometrical data of the virtual mandibular resections and of the stereolithographic model are used to choose the ideal flap and to contour the flap in three planes into an ideal, best-fit neo-mandible for accurate setting into the resection site.

Once the bone flap is harvested and contoured, its setting into the defect is summarised as follows:

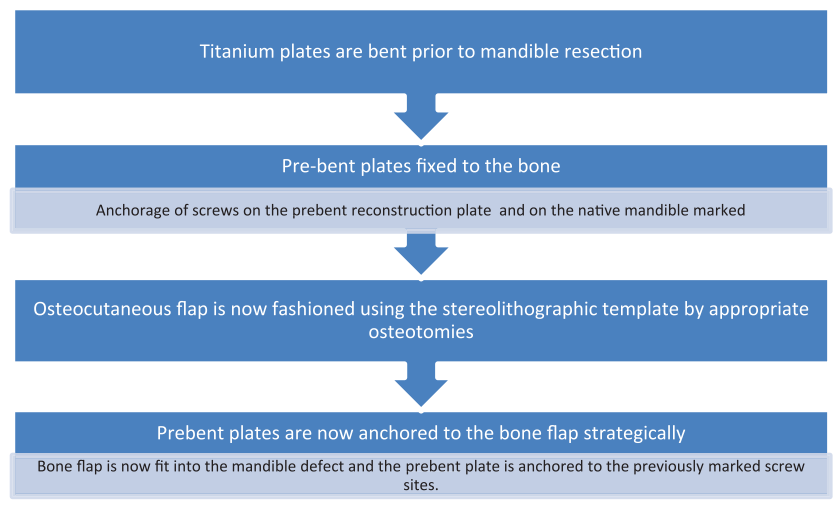

Anastomosis of vessels is then completed and occlusion checked. Pantomographies are performed immediately after surgery and at the end of the bone consolidation period (3 months after surgery).

The following are the clinical situations where we have used stereolithographic models:

\section{Case 1}

A young girl presented with a hypoplastic left hemimandible. She was planned for a hemimandible reconstruction using free fibula osteocutaneous flap. A $3 \mathrm{D}$ model of the mandible was constructed using the mirror image principle. The two models were then split in the midline and the two normal halves were joined in the middle, while maintaining the correct intercondylar distance. This model was then used as a guide to pre-bend the reconstruction plate and contour the harvested fibula. The fibula was harvested and osteotomised accordingly, anchored with pre-bent reconstruction plates. Preoperative and postoperative photographs are shown for comparison and also shown are pictures of the fibula designed as per the model [Figures 2 and 3].

\section{Case 2}

Primary mandible reconstruction for carcinoma: A 50-yearold gentleman presented with carcinoma of anterior floor of the mouth with central segment mandible involvement. The preoperative photograph of the chin and submandibular

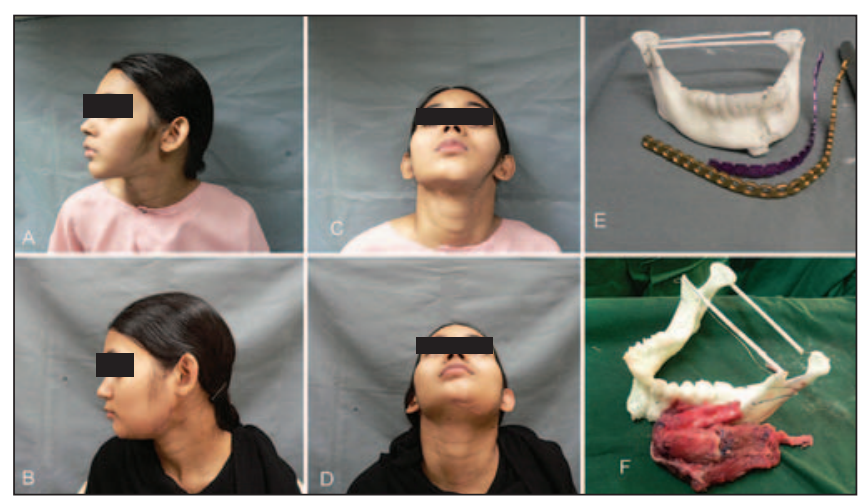

Figure 2: Case 1- Young girl with left hypoplastic mandible secondarily reconstructed using stereolithographic model. (A \& B) Pre and Post operative lateral views. (C \& D) Pre and Post operative worm's eye views (E) Stereolithography 3D model with pre-bent plate (F) Shaping of the fibula in accordance with the 3D model

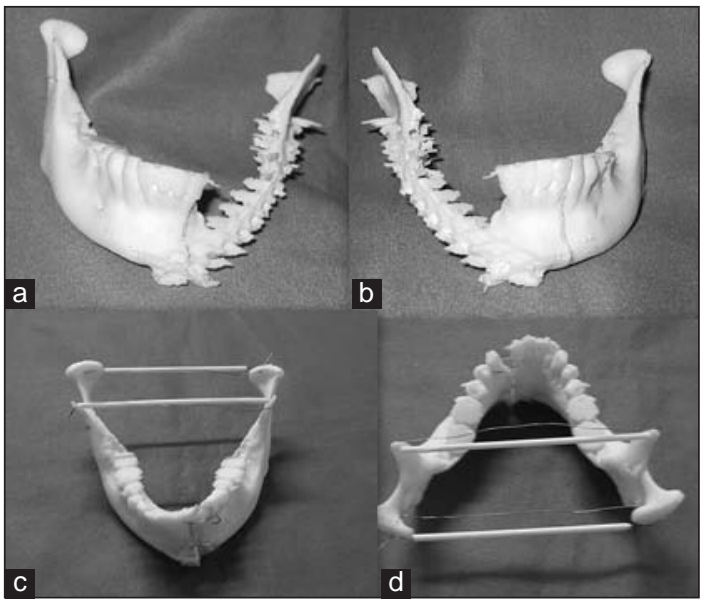

Figure 3: Case 1- Mirror image used to reconstruct the entire normal mandible (a) Right normal hemi-mandible model (b) Left hemi-mandible model created using mirror image principle (c) Both the halves joined to form the entire normal mandible, determined by the intercondylar distance (d) Stereolithography: complete mandible model 
region is shown along with the corresponding CT image. A 3D stereolithographic model was prepared using CAD. This model was used to osteotomise the free fibula and the reconstruction plate bent accordingly off table, while the tumour was being resected simultaneously. The donor site was closed primarily. This greatly helps in reducing operative time and avoiding contamination. The defect in situ is shown along with the inset of the osteotomised fibula graft. Six months' follow-up of this patient is shown [Figures 4 and 5].

\section{Case 3}

A 35-year-old patient presented with carcinoma left

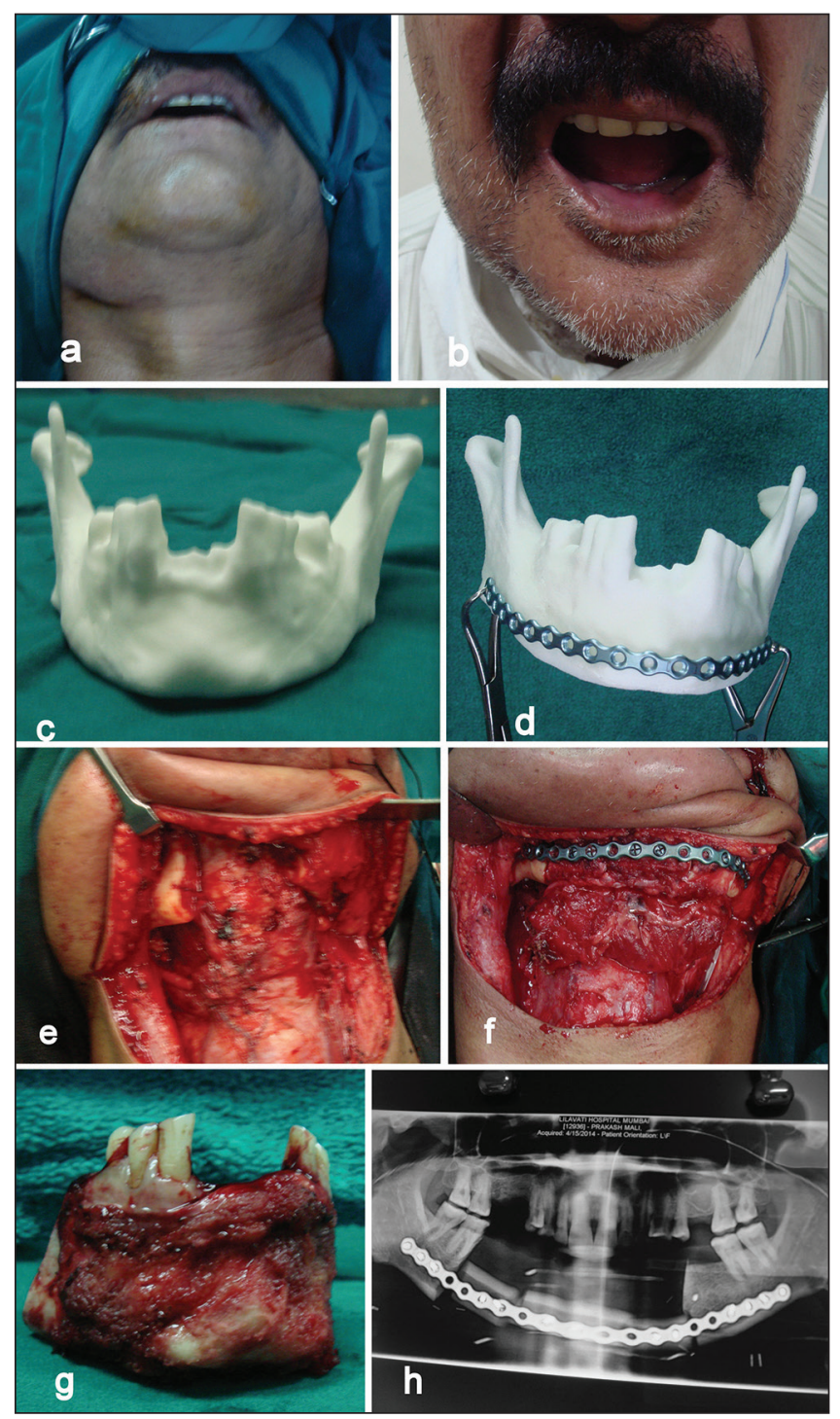

Figure 4: Case 2- Primary mandible reconstruction in a patient with floor-of-themouth cancer and central segment mandible involvement (a) Preoperative picture with central segment mandible involvement (b) Postoperative picture (c) Mandible 3D model (d) Pre-bent plate moulded according to the mandible (e) Central segment mandible resection (f) Central segment mandible reconstruction done with fibula flap (g) Resected specimen (h) Postoperative pantomogram maxilla. Using a sterolithographic maxillary model, the tumour was resected on the model and the requisite bone graft was planned and designed. A vascularised iliac crest free bone flap was then contoured using the stereolithography template of the maxillary defect. A postoperative photograph of the patient is shown [Figure 6].

\section{Case 4}

A 40-year-old patient presented with a discharging sinus due to infected implants following a non-vascularised bone graft attempted elsewhere. Her secondary reconstruction was possible using the mirror image principle and a normal mandibular model was thus created. Reconstruction proceeded with free fibula vascularised bone flap [Figures 7 and 8].

\section{Case 5}

Secondary reconstruction with spanning plate in situ was done for the left hemi-mandible in an 18-year-

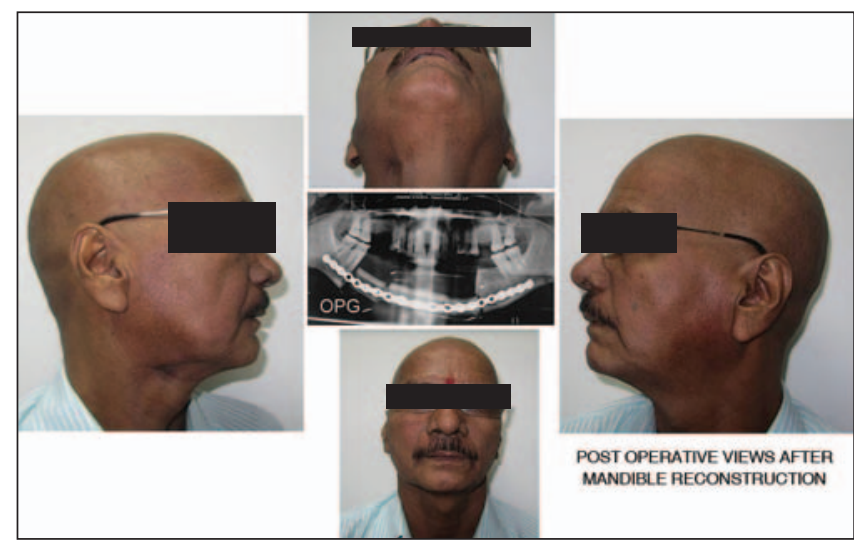

Figure 5: Case 2- Postoperative views of patient showing well-defined mandible contours of lower border and satisfactory projection

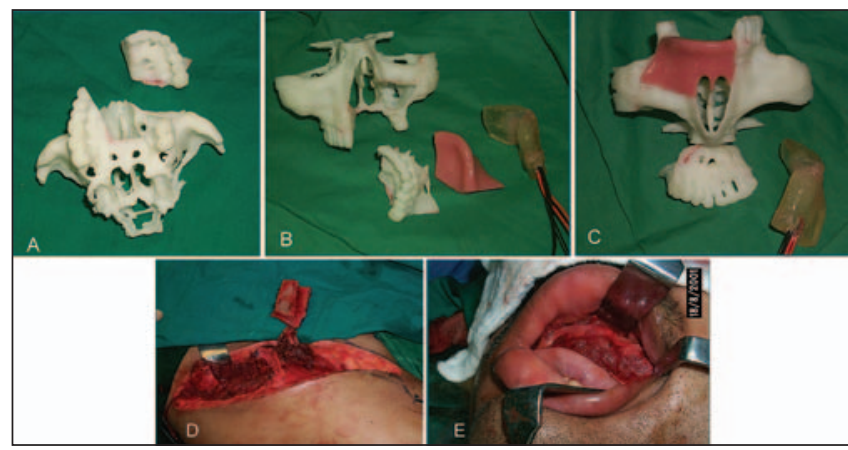

Figure 6: Case 3- Template for resection and reconstruction and orienting aid during surgery (a) 3D stereolithography model with model of resected specimen (b) Model of bone to be reconstructed and bone graft model (c) Bone graft model fit onto the defect on the model to check conformation (d) Iliac crest vascularised bone graft harvested according to the model (e) Bone graft inset into the maxillary defect 
old girl. A vascularised iliac crest flap was used and designed according to the template to ascertain the exact shape of the angle of the mandible [Figures 9 and 10$]$.

\section{Case 6}

Stereolithography was used to reconstruct a posttraumatic right-sided fronto-temporal bony defect using an implant. The implant was designed using stereolithography. The entire skull with the defect as well as the corresponding implant to fill the defect was constructed using CAD. A custom prosthesis was fabricated to exactly fit into the bony defect and the model was used to confirm its exact fit. Anchorage of screws to fix the custom prosthesis 'on the bench' was done and then the prosthesis with implants was fixed

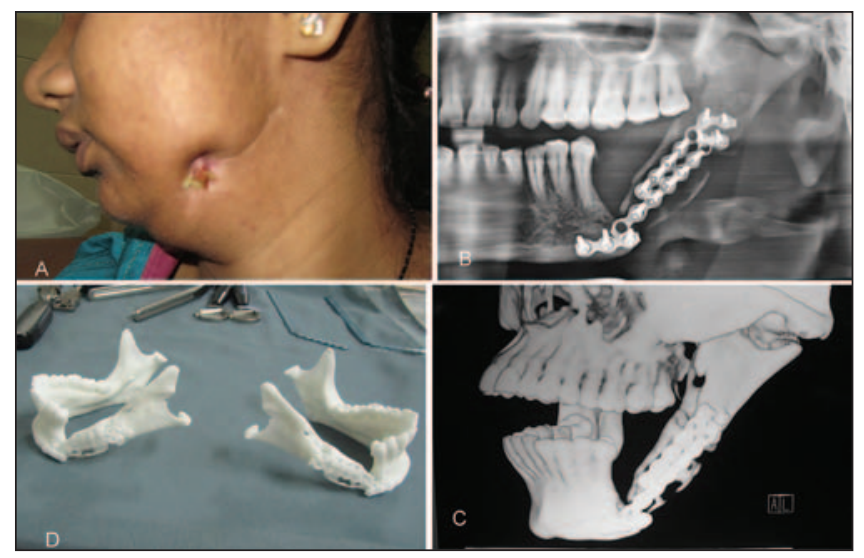

Figure 7: Case 4- Secondary mandible reconstruction for previous suboptimal reconstruction: Presentation and planning (a) Patient presenting with discharging sinus due to infected implants (b) Pantomogram revealing the affected left hemi-mandible with implants (c) CT image of the affected left hemi-mandible (d) Sterolithography model of normal left hemi-mandible constructed using lateral imaging principle

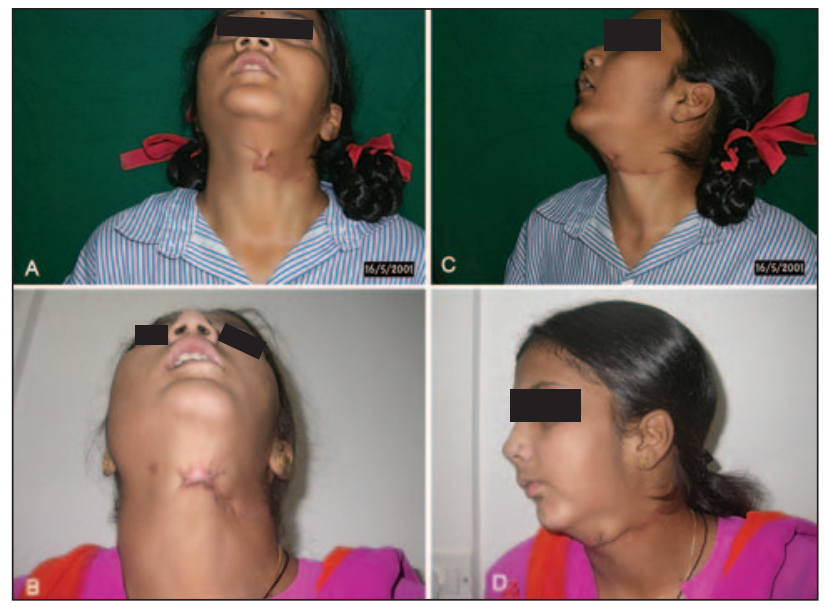

Figure 9: Case 5- Secondary mandible reconstruction of left hemi-mandible $(A \& B)$ Pre and post operative worm's eye views (C \& D) Pre and Postoperative lateral views to the skull defect [Figures 11-13]. The preoperative and postoperative photographs show a satisfactory $3 \mathrm{D}$ reconstruction.

\section{Case 7}

A 45-year-old patient presented for a secondary reconstruction of the left hemi-mandible. She had undergone left hemi-mandibulectomy approximately 8 years ago and pectoralis major myocutaneous flap was used for reconstruction followed by radiotherapy. She presented with trismus, with mouth opening of $1 \mathrm{~cm}$ and obliteration of the left-sided gingivolabial sulcus extending across the midline. The mandibular defect crossed the midline. As the entire condyle was absent, a sterolithographic model was constructed based on lateral imaging and using the distance between the

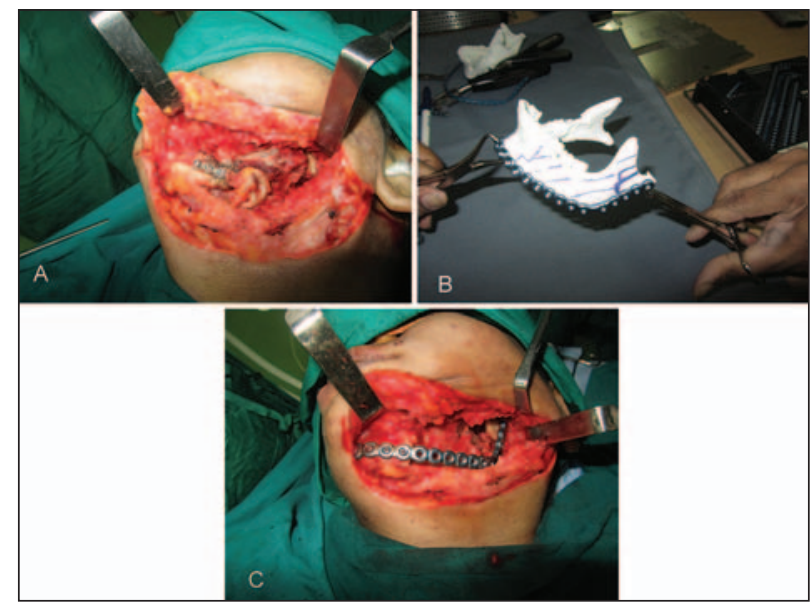

Figure 8: Case 4- Secondary mandible reconstruction for previous suboptimal reconstruction, intraoperative procedure (a) Infected implants seen with residual mandibular tissue (b) Reconstruction plate bent according to the stereolithography model (c) Anchoring of reconstruction plate after defect creation

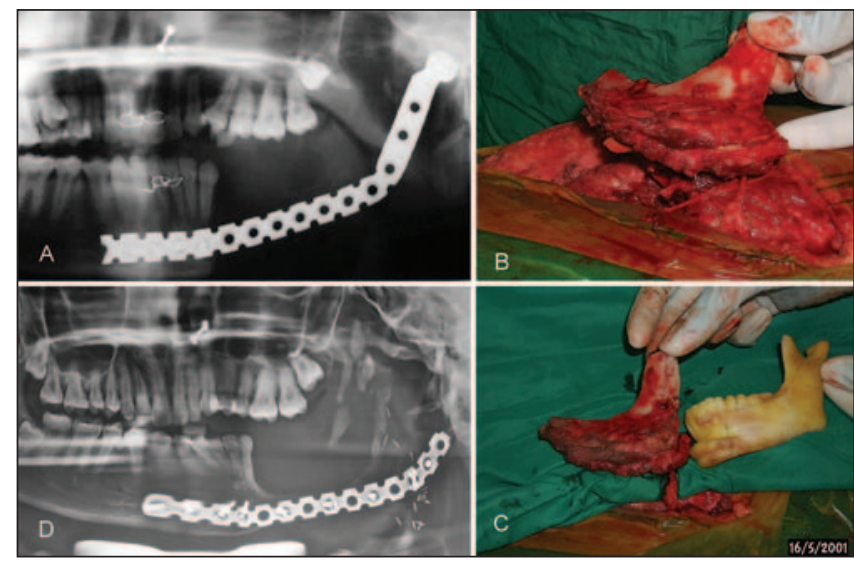

Figure 10: Case 5- Improved surgical planning using stereolithography (a) Pantomogram showing reconstruction plate spanning the left hemi-mandible defect (b) Harvest of vascularised iliac crest flap (c) Shaping of the iliac crest flap in accordance with the model (d) Postoperative pantomogram showing vascularised bone in situ 


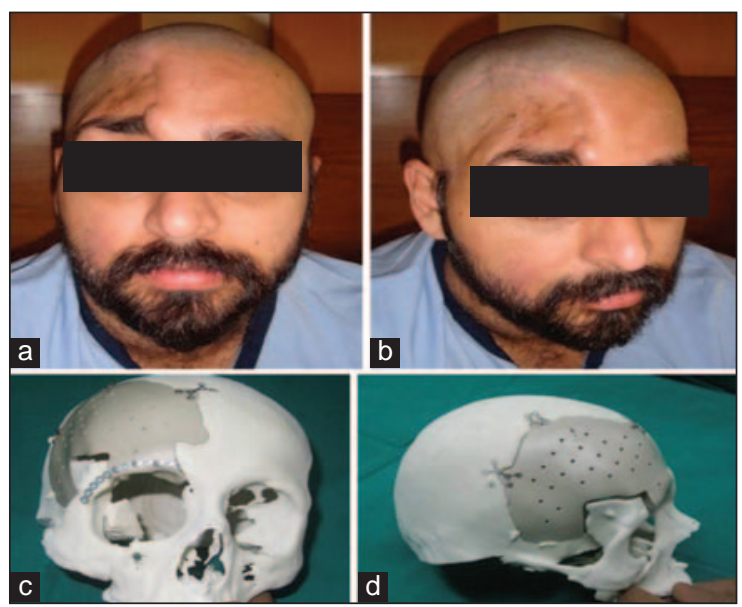

Figure 11: Case 6- Production of surgical implant for secondary skul reconstruction (a) Frontal view showing the right post-traumatic frontotemporal defect (b) Closer view of the skeletal defect (c) Stereolithographic model constructed of the skull with the defect (d) Patient-specific PEEK(polyetheretherketone) implants customised as per the stereolithographic model, with pre-determined points of anchorage
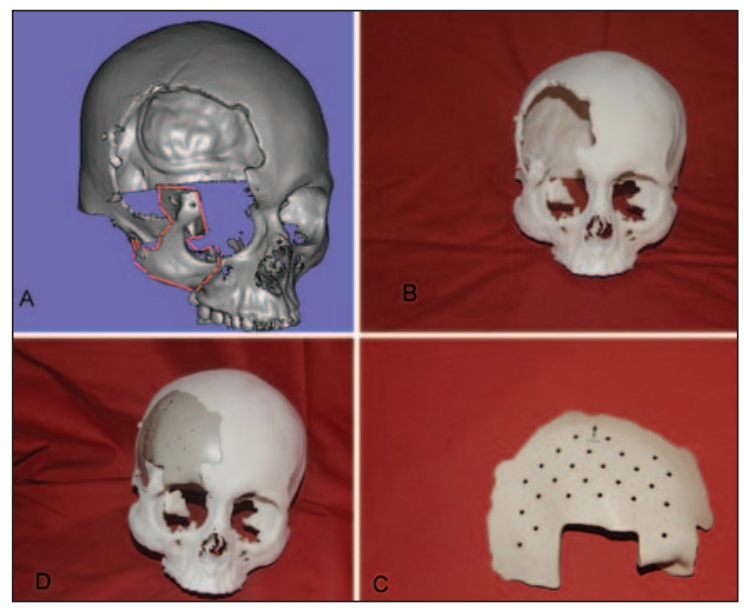

Figure 12: Case 6- Model production (a) CAD using MIMICS (b) Construction of skull model (c) Construction of implant template (d) Conformation of implant into the defect
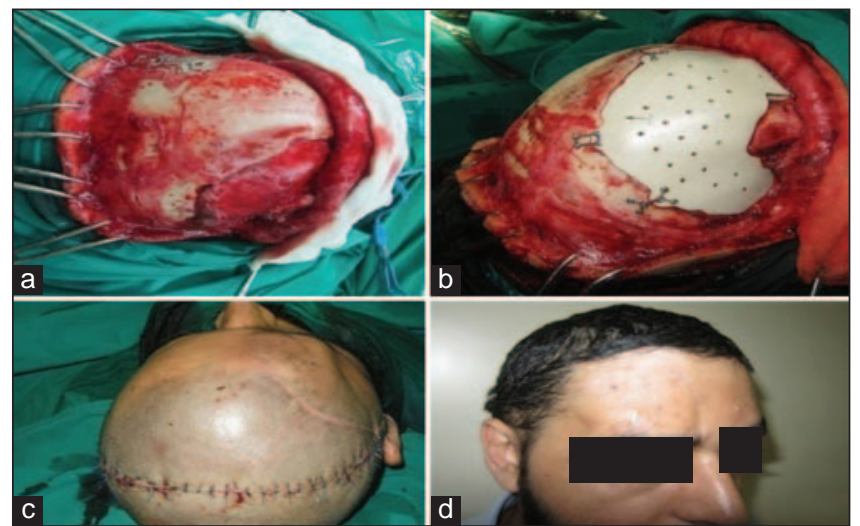

Figure 13: Case 6- Preoperative simulation using stereolithography

(a) Intraoperative view of the skull defect (b) Implant anchored onto the defect with perfect conformation (c) Immediate postoperative view (d) Late postoperative view showing symmetric facial contours two glenoid fossae. Surgery was commenced with creation of the true defect and release of trismus with creation of sulcus. A free fibula flap was shaped with two osteotomies and pre-bent plate contoured onto the bone flap. The bone flap was anchored, followed by inset of skin paddle to create a sulcus all along. Anastomosis was done using contralateral neck vessels after passing the pedicle through a tunnel between the native bone and digastric muscle. The patient's occlusion was satisfactory, while the trismus did not improve significantly despite doing a right-sided coronoidectomy [Figures 14 and 15].

\section{Case 8}

A 35-year-old female presented with ameloblastoma of the right hemi-mandible. She underwent tumour excision in the form of hemi-mandibulectomy with primary mandibular reconstruction using free fibula vascularised bone. Stereolithographic model was used to shape the hemi-mandible and the end result was satisfactory [Figures 16 and 17].

\section{Case 9}

Secondary right hemi-mandible reconstruction for a male patient aged 50 years: He had undergone right hemi-mandibulectomy about 7 years ago, and presented with retrognathia and malocclusion. Right hemimandible reconstruction was done using a stereolithographic model, with correction of occlusion to class 1 [Figures 18 and 19].

\section{DISCUSSION}

The construction of accurate anatomical replicas using digital design and manufacturing techniques can be an extremely useful method for employing imaging studies by transforming data into tangible objects. ${ }^{[6]}$ For tumour surgery and reconstruction, the ability to manipulate the 3D images with computer software is very helpful in recreating the symmetry of the jaw after resection. ${ }^{[7]}$ Rose et al. first reported the use of high-tech $3 \mathrm{D}$ computer-generated models in facial reconstructions with vascularised grafts. ${ }^{[8]}$

Rapid prototyping-generated anatomical models translate image data into solid replicas, giving surgeons the means for tactile interaction with patient anatomy prior to an operation. 


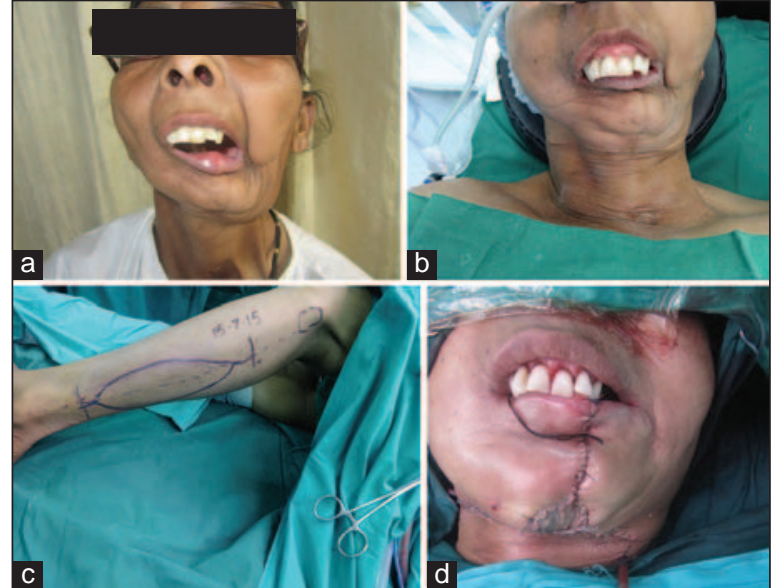

Figure 14: Case 7- Secondary reconstruction of left hemi-mandible for left hemi-mandibulectomy done 9 years ago followed by radiotherapy (a) Left hemi-mandibulectomy defect showing deviation of mouth (b) Retrognathic mandible with severe trismus and evasion of sulci (c) Left fibula flap harvested (d) Postoperative reconstructed mandible
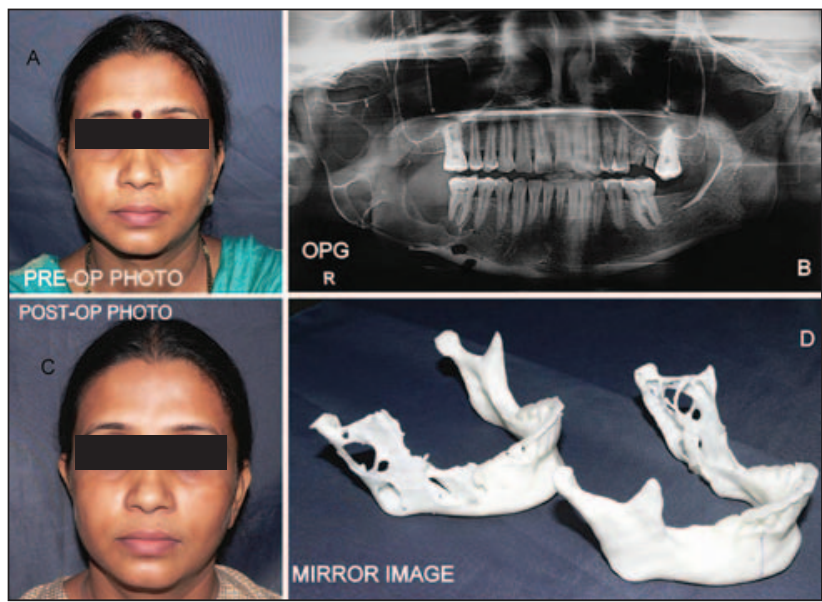

Figure 16: Case 8- Right hemi-mandible ameloblastoma (a) Preoperative photograph (b) Pantomogra showing lytic lesions of ameloblastoma (c) Postoperative photograph showing satisfactory restoration of contours (d) Stereolithographic model of left normal hemi-mandible and construction of right normal hemi-mandible using mirror image principle

Applications of stereolithography: PRIMARY RECONSTRUCTION

- With deformed jaw

- With normal jaw

SECONDARY RECONSTRUCTION

- With spanning plate

- With gap

In secondary cases, the native hemi-mandible or segment is not available. Hence, the first requirement is to have a model of that which needs to be reconstructed. This is obtained from the non-affected side of the mandible by the principle of lateral imaging. The CAD data of
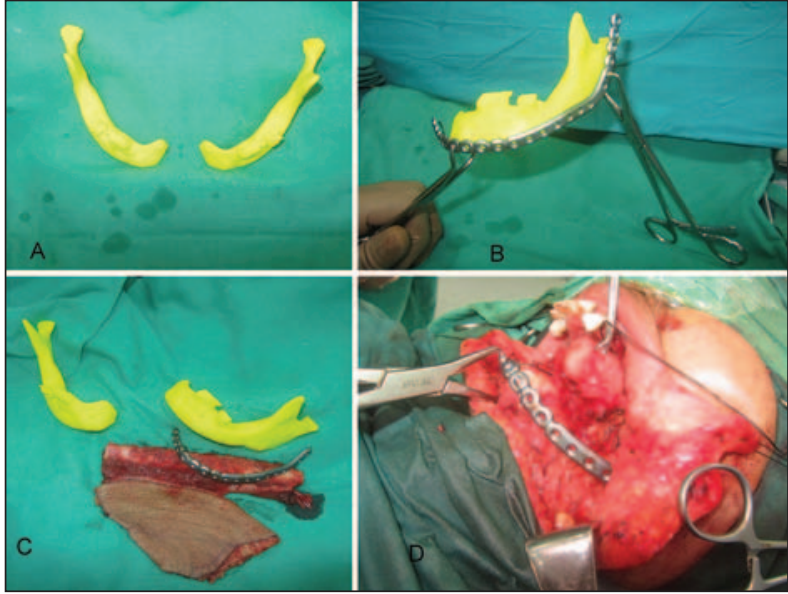

Figure 15: Case 7-Improved surgical planning using stereolithography (a) Left hemi-mandible constructed using lateral imaging principle (b) Plate shaped according to mandible model (c) Fibula harvested and ready to be shaped (d) Plate anchored after creation of true defect
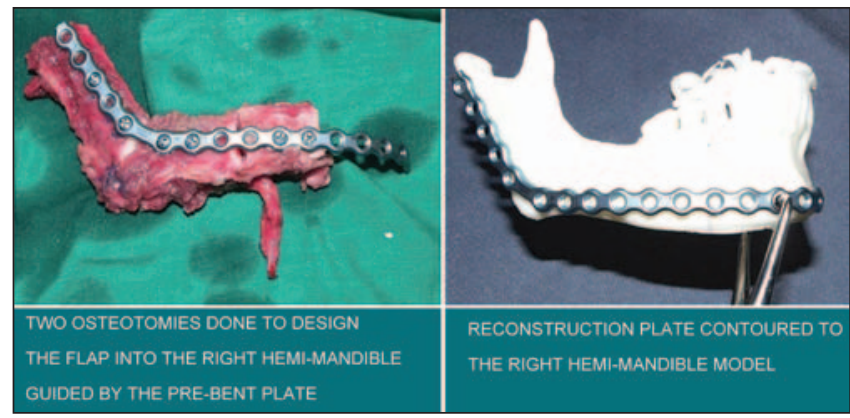

Figure 17: Case 8- Reduced surgical time with the aid of stereolithographic model (a) Free fibula flap osteotomised according to the model with anchorage of plate off table (b) Contouring of reconstruction plate off table

the normal hemimandible is used to manufacture the affected (distorted/deformed) hemi-mandible by lateral imaging (mirror image principle). Thus this mirror image produced helps in creating the mandible 3D model. This aids in accurate assessment of the true defect, as the apparent defect may be much smaller because of fibrosis, contracture and scarring which accompany secondary reconstruction. Thus, the same data and CAD can be used to produce the entire normal mandible. In cases where a patient presents with hemi-mandibulectomy beyond the midline with intact condyle, the two halves are derived as described above. To ascertain the midline of the mandible, the intercondylar distance is used. In cases of hemi-mandibulectomy beyond the midline with absent condyle, the glenoid fossae are used to calculate the intercondylar distance, and the midline of the mandible can then be determined.

In cases of secondary reconstructions in patients with malocclusion, this model reconstruction of both jaws 


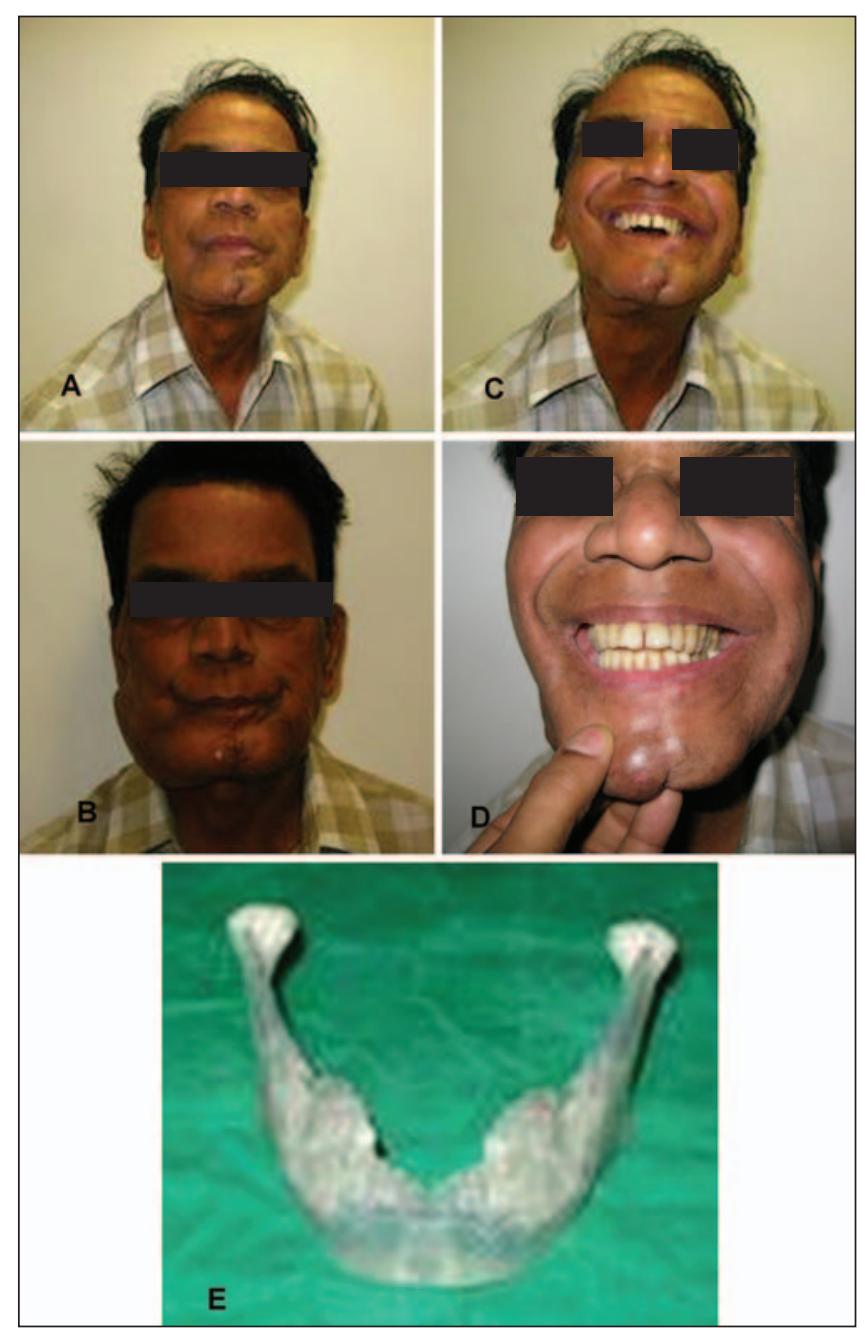

Figure 18: Case 9- Secondary mandible reconstruction (a) Preoperative photograph (b) Preoperative malocclusion with retrognathia (c) Postoperative photograph showing contour correction (d) Postoperative occlusion, class 1

helps in assessing the occlusal deformity and to prepare occlusal splints to correct occlusion (this is taking things a step further than dental immersions).

Shaping of the mandible in the past has been attempted such as by the following methods:

1. Bending the plates/wires by trial and error.

2. Acrylic templates of patients have been studied and mean of the angles of mandibles and the length of its various segments have been assessed. Based on this, a standardised pre-fabricated model was used in the past.

3. Virtual plate bending: Using MIMICS software, virtual resection, virtual simulation and virtual pre-bending of the titanium plates can be done. ${ }^{[9]}$

4. Computer-assisted mandible reconstruction (CAMR): Here, fibula cutting guides are placed at the cut ends of the fibula to replicate cuts of both the end and closing wedge osteotomies. ${ }^{[10]}$

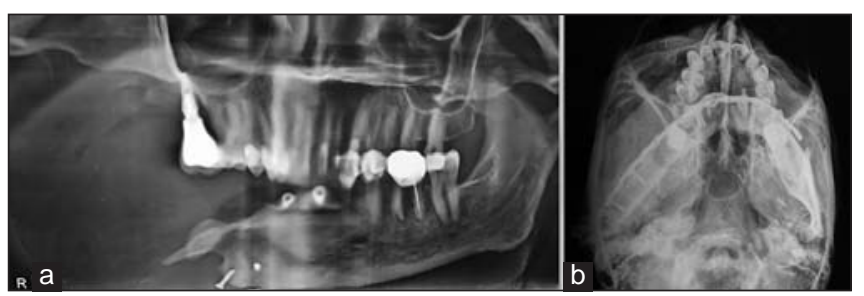

Figure 19: Secondary mandible reconstruction (a) Preoperative pantomogram showing right hemi-mandibulectomy (b) Postoperative radiograph showing hemi-mandible reconstruction

Models can be highly effective for surgical planning, improving communication between medical experts and patients, and facilitating customisation of treatment devices:

- To aid production of surgical implants

- To improve surgical planning

- To act as an orienting aid during surgery

- To enhance diagnostic quality

- To use in preoperative simulation

- To easily explain to patients the nature of the surgery and the surgical plan

- To prepare a template for resection

3D models can be used to pre-shape/pre-bend metallic implants that will be used in surgery. The length of implant plates and the number and lengths of the required screws can be planned before surgery. ${ }^{[11]}$ It is also possible to plan osteotomies by performing a surgical rehearsal on the model itself. Such a rehearsal allows measurement of the displacement of all bone segments and anticipation of the size and the form of bone grafts. ${ }^{[1]}$

The models clearly show deformities or disease states and provide the surgeon with a mental image of the patient's anatomy that, in turn, improves the accuracy of plate and screw placement. ${ }^{[12]}$ Adapting a reconstruction plate intraoperatively, prior to tumour resection, would interfere with radical surgery, ${ }^{[13]}$ but it can be easily adapted on the table to the template and then its conformation can be checked on the native mandible just prior to resection. We found that operating time was reduced by about $1-1.5 \mathrm{~h}$ in comparison to performing the surgery without a model.

The virtual model and its planning act as a guide to determine the length, the shape (in particular the mandible angle), the height and the contour of the bone graft.

Till 2009, the cost of these 3D printers continued to be high as they were mainly for industrial purposes. From 2009, commercial low-price 3D printers $(<10,000$ USD) 
started being developed. From 2012, FDM technology using polylactic acid became the most common 3D printing technology used for commercial printers. ${ }^{[14]}$

Though this technology has been used around the world for the last 30 years, it was not used much in India until recently due to the following reasons:

1. High costs of the printers and material, which could not be afforded by most centres in India.

2. Lack of technical aid for data conversion and model manufacturing.

3. Costs of manufacturing and shipping models from the West, which would have been unreasonably high.

4. Lack of awareness on the applications of 3D printing in the field of reconstruction.

\section{The future}

With the availability of low-cost commercial 3D printers and desktop 3D printers along with the material, 3D printing can be well-utilised for several applications, depending on the surgeons' imagination.

\section{To name a few:}

1. Excision of head and neck tumours with precision and avoiding any untoward complications. This also helps in a planned reconstruction.

2. Excision of bone tumours and precise osteotomy of bone grafts for faster healing and weight bearing.

3. Soft-tissue reconstruction can be aided by 3D printing to obtain the precise contour and volume of soft tissue required for reconstruction as in hemi-facial atrophy or soft-tissue tumours of face.

4. 3D printing has been described for understanding perforator anatomy.

5. It may help in predicting post-surgery appearances.

\section{CONCLUSION}

The advantages of stereolithographic models include decreased surgical time, preoperative planning, prebending implants, surgical rehearsal, patient education and, ultimately, more predictable surgical results of occlusion. It helps in improving the aesthetics of standard reconstructive procedures.

\section{Declaration of patient consent}

The authors certify that they have obtained all appropriate patient consent forms. In the form the patient(s) has/have given his/her/their consent for his/her/their images and other clinical information to be reported in the journal.
The patients understand that their names and initials will not be published and due efforts will be made to conceal their identity, but anonymity cannot be guaranteed.

\section{Acknowledgement}

We would like to thank Mr. Paresh Bheda, Protosys Technologies Pvt. Ltd., for constructing the models and for providing us with an understanding of the process of stereolithography.

\section{Financial support and sponsorship \\ Nil.}

\section{Conflicts of interest}

There are no conflicts of interest.

\section{REFERENCES}

1. D'souza KM, Aras MA. Applications of computer-aided design/ computer-assisted manufacturing technology in dental implant planning. J Dent Implant 2012;2:37-41.

2. VoitikAJ. CT data and its CAD and CAM utility in implant planning: Part I. J Oral Implantol 2002;28:302-3.

3. Gibson I, Rosen DW, Stucker B. Additive Manufacturing Technologies. Springer; 2010. p. 238.

4. Winder J, Bibb R. Medical rapid prototyping technologies: State of the art and current limitations for application in oral and maxillofacial surgery. J Oral Maxillofac Surg 2005;63:1006-15.

5. Mankovich NJ, Cheeseman AM, Stoker NG. The display of threedimensional anatomy with stereolithographic models. J Digit Imaging 1990;3:200-3.

6. Jones R, Haufe P, Sells E, Iravani P, Olliver V, Palmer C, et al. RepRap - the replicating rapid prototyper. Robotica 2011;29: 177-91.

7. Brown GA, Milner B, Firoozbakhsh K. Application of computergenerated stereolithography and interpositioning template in acetabular fractures: A report of eight cases. J Orthop Trauma 2002;16:347-52.

8. Powers DB, Edgin WA, Tabatchnick L. Stereolithography: A historical review and indications for use in the management of trauma. J Craniomaxillofac Trauma 1998;4:16-23.

9. Rose EH, Norris MS, Rosen JM. Application of high-tech three dimensional imaging and computer-generated models in complex facial reconstruction with vascularized bone grafts. Plast Reconstr Surg 1993;91:252-64.

10. Rahimov $C$, Farzaliyev I. Virtual bending of titanium reconstructive plates for mandibular defect bridging: Review of three clinical cases. Craniomaxillofac Trauma Reconstr 2011;4:223-34.

11. Succo G, Berrone M, Battiston B, Tos P, Goia F, Appendino P, et al. Step-by-step surgical technique for mandible reconstruction using free fibula flap: Application of digital technology in virtual surgical planning. Eur Arch Otorhinolaryngol 2015;272: 1491-501.

12. Lal K, White GS, Morea DN, Wright RF. Use of stereolithographic templates for surgical and prosthodonticimplant planning and placement. Part I. The concept. J Prosthodont 2006;15:51-8.

13. Chang PS, Parker TH, Patrick CW Jr, Miller MJ. The accuracy of stereolithography in planning craniofacial bone replacement. J Craniofac Surg 2003;14:164-70.

14. Cunningham LL Jr, Madsen MJ, Peterson G. Stereolithographic modeling technology applied to tumor resection. J Oral Maxillofac Surg 2005;63:873-8. 